\title{
Brain Death Determination: Still a Lot to Learn, Still a Lot to Do...
}

\author{
Panayiotis Varelas
}

Published online: 15 October 2014

(c) Springer Science+Business Media New York 2014

Human life is book-ended by two major events, birth and death. Although all are born the same way, since the invention of ventilators and ICUs humans can die either by cardiorespiratory arrest or by irreversible cessation of all brain functions [the so-called death by neurological criteria or "brain death (BD)"]. Diagnosing the former is an easy process requiring an electrocardiogram or a stethoscope. Diagnosing the latter, however, is a complex process that requires experience. There is wide-spread variability across countries or across states and hospitals in the US on how patients are pronounced BD [1]. There are also many unanswered questions regarding the BD process and, because of the lack of high-level evidence, continuous challenge of its core notions by a substantial minority of physicians. In fact, few years ago, when the American Academy of Neurology BD Guidelines were revised, and a panel of experts attempted to answer five basic questions, they concluded that in 4/5 questions the level of evidence was $\mathrm{U}$ (data inadequate or conflicting) and in 1/4 level $\mathrm{C}$ (possibly effective, ineffective, or harmful for the given condition in the specified population) [2]. Therefore, any attempts to conduct quality research and answer questions pertaining to $\mathrm{BD}$ are more than welcomed. In this issue of Neurocritical Care, our readers will find four studies that try to address four different BD problems.

In the first paper Citerio et al. [3], examined the variability in BD determination in 28 European countries that responded to a survey. Across the Atlantic, variability occurs in State laws, hospital policies, bedside performance of physicians, and documentation of BD [1, 4]. Therefore, it is no surprise to report that variability also exists across European

P. Varelas $(\square)$

Henry Ford Hospital, Detroit, USA

e-mail: PVARELA1@hfhs.org countries. Basic prerequisites such as normal core temperature or blood pressure were not required in one and ten countries, respectively. Clinical exam alone was sufficient in $50 \%$ of countries and in the rest some ancillary test was required. Important parts of the clinical exam, such as the gag reflex, were not defined in six countries and the apnea test details in four. The number of clinical exams varied from 1 to 3 and the time interval between exams from no minimum to $12 \mathrm{~h}$. The list can go on, but the reader, like the authors, can easily conclude one of two things: that an international consensus on BD determination is imperative and that some clinical competency requirement [5] or check lists (like those used in the operating room or the one provided by the AAN [2]) may put further order into an already chaotic situation.

One step in the right direction is the paper by MacDougall et al. [6]. The authors describe a BD didactic course and simulation exercise that they developed at Yale. A set of questions was administered before the didactic session and after the simulation. The authors report that there was a significant improvement in responses, which initially were correct in only $41.5 \%$ of the time. Interestingly, even neurointensivists (who ostensibly are performing $\mathrm{BD}$ determinations regularly) answered correctly no higher than $56.6 \%$ of the time in the pre-test. Attendings in neurology or neurosurgery scored higher in both pre-test and the 26-point clinical exam than other specialties. The authors should be commended for this effort. Although there was no scripted mention of coma in a patient to be examined for BD and their course is a rather expensive approach (not all hospitals have manikins with pupillary reactivity capability!), this is an excellent initial effort that could be easily adopted and customized widely. Long-term effect of this intervention should also be assessed in the future, since lack of frequent real-life 
practice of BD exams may lead to a "fade away" skill phenomenon.

One of the most potent indicators of BD is lack of breathing during an acute hypercarbic stimulus (the positive apnea test). Because the oxygenation-diffusion method may lead to hemodynamic instability due to the acute respiratory acidosis (hypotension, cardiac arrhythmias), pneumothorax or hypoxia, many physicians have concerns about performing the test. These are refuted by Datar et al. [7], who retrospectively evaluated 63 patients at the Mayo Clinic. Only one patient had the test aborted due to desaturation, but the starting point for this particular patient was high $\mathrm{FiO} 2$ and PEEP. Mild hypoxemia was documented in four other patients $(6.3 \%)$ and an easily controlled hypotension in another $11(17.4 \%)$. No patient developed arrhythmias or cardiac arrest. This is an improvement compared to an older and larger cohort from the same institution, with fewer patients excluded from the apnea testing and fewer aborted tests. The increased involvement of neurointensivists in the vast majority of this testing in the current study may be one explanation for the lower failure to initiate or complete the test, as the authors suggest. If such an association is proven, this will be another weapon in the proponents' armamentarium for BD standardization or BD certification or performance of the exam by a small and focused group of physicians (neurointensivists?) who practice it frequently.

Ancillary tests were initially used as diagnostic tests for BD and later found their place in confirming BD in case the clinical exam or the apnea could not be completed. Some authorities suggest these tests have no place in modern practice and a carefully executed clinical BD evaluation following the AAN Guidelines is sufficient [8]. There are, however, situations where a complete exam cannot be completed (for example, facial trauma precluding evaluation of cranial nerves) and an ancillary test in most hospital policies is either mandatory or simply an alternative. Computed tomography angiography (CTA) is less invasive than conventional 4-vessel angiogram. It is also more available in most hospitals compared to digital subtraction angiography (DSA) or radionuclide imaging (RNI), which requires more specialized personnel and equipment. The problem with CTA is that there are no uniform criteria for evaluating the absence of intracranial flow. In the most recent AAN Guidelines, CTA was one of the newer ancillary tests that were evaluated, with the conclusion being that there is insufficient evidence for its use to confirm BD [2]. In this issue of our journal, Kramer and Roberts [9] report the results of a systematic review of CTAs for this purpose, using specific literature search criteria and comparing it to either a clinical exam as "gold standard" for BD or absent flow by DSA or RNI. They eventually included 12 studies and they report that depending on the diagnostic criteria of arrested intracranial flow used, the sensitivity of the test ranged from 62 to $99 \%$. The highest sensitivity was achieved when the absence of opacification of the internal cerebral veins was used as a criterion. The authors were not able to report specificity, which is problematic for a test that ideally should have no false positives (positive test for BD when the patient in reality is not). Their conclusion is similar to the AAN Guidelines, in that this test should not be recommended until studies with more defined criteria and better validation are published.

What should the interested reader learn from this new information? The skeptics will find new ammunition against the notion of $\mathrm{BD}$, but for the majority of us who accept it, several conclusions can be reached. BD declaration is not simple and the unacceptable variability between countries, states, hospital policies, and individual practitioners should end. This Babel tower just undermines the trust of the general public, induces confusion in health care providers and creates medico-legal nightmares. The need for an integration of laws and policies at the level of the European Community becomes obvious from the $\mathrm{Ci}$ terio et al., paper and could establish a paradigm to follow for the big democracies across the Atlantic and beyond. The Yale paper, the first of its kind, shows that simulation workshops for $\mathrm{BD}$ is one fast, tangible solution to the problem compared to legislative and regulatory endeavors, which may take years or decades to materialize.

Of narrower scope, the other two papers deal with the BD declaration process and answer important questions on what an apnea test entails and if CTA should become mainstream. There are few interesting points from these two papers. First, complications are rare and have become even rarer as more experts in BD determination weight in. However, the apnea test period is not a time to relax. If the patient is not already on pressors, it would be advisable to have an infusion of phenylephrine or norepinephrine ready to go as the blood pressure slowly decreases. Equally important is vigilance for desaturations, which can occur quickly. In such a case, one needs to be ready to draw blood gases fast and reconnect the patient to the ventilator. Special attention should be paid to the oxygenation tubes that are inserted through the endotracheal tube and the oxygen flow level that is administered. They should be smaller in diameter than the endotracheal tube in order to allow unobstructed flow of oxygen in and out. I have personally witnessed pneumothorax from a high oxygen flow and I seldom use flows higher than 6-7 L/min. Second, despite the fact that CTA is widely available as an ancillary test, the exact details on what one should look for have not yet been determined. Apparently, lack of the deep venous system opacification seems more promising than the complete absence of intracranial arterial flow. A 
broader discussion, if and when to use these tests for BD confirmation, although touched by Kramer and Roberts' paper, was not explored and should be the basis for future research. In the technological and fast-paced era we live in, one may question the use of clinical exams to confirm the presence of irreversible cessation of all brain functions and rely more on flow tests to even bypass prerequisites for $\mathrm{BD}$ (such as the still ill-defined toxometabolic derangements). There are problems with this approach, however, ranging from the inconsistent criteria used to the absence of continuous monitoring of cerebral blood flow (or the lack of) for a long enough period to confirm functional and structural irreversibility in the brain integrator areas.

All these unsolved issues beg for more standardization and more research. We will need more level A evidence after we reach the point when BD determination is finally as easy and convincing as putting a stethoscope to one's chest to search for a heartbeat and a breath that will never come.

\section{References}

1. Greer DM, Varelas PN, Haque S, Wijdicks EF. Variability of brain death determination guidelines in leading US neurologic institutions. Neurology. 2008;70:284-9.
2. Wijdicks EF, Varelas PN, Gronseth GS, Greer DM. Evidencebased guideline update: determining brain death in adults: report of the Quality Standards Subcommittee of the American Academy of Neurology. Neurology. 2010;74:1911-8.

3. Citerio G, Crippa IA, Bronco A, Vargiolu A, Smith M. Variability in brain death determination in Europe: looking for a solution. Neurocrit Care. 2014. doi:10.1007/s12028-014-9983-x.

4. Shappell CN, Frank JI, Husari K, Sanchez M, Goldenberg F, Ardelt A. Practice variability in brain death determination: a call to action. Neurology. 2013;81:2009-14.

5. Bartscher JF, Varelas PN. Determining brain death-no room for error. Virtual Mentor. 2010;12:879-84.

6. MacDougall BJ, Robinson JD, Kappus L, Sudikoff SN, Greer DM. Simulation-based training in brain death determination. Neurocrit Care. 2014. doi:10.1007/s12028-014-9975-x.

7. Datar S, Fugate J, Rabinstein A, Couillard P and Wijdicks EFM. Completing the Apnea test: decline in complications. Neurocrit Care. 2014. doi:10.1007/s12028-014-9958-y.

8. Wijdicks EF. The case against confirmatory tests for determining brain death in adults. Neurology. 2010;75:77-83.

9. Kramer AH, Roberts DJ. Computed tomography angiography in the diagnosis of brain death: a systematic review and metaanalysis. Neurocrit Care. 2014. doi:10.1007/s12028-014-9997-4. 